\title{
The Contamination of Substorm Fields by Induction Effects: Extension to the Heterogeneous Surface Layer
}

\author{
Marianne MARESCHAL* and Guy VASSEUR \\ Centre Geologique et Geophysique, USTL, Pl. Bataillon, 34060 Montpellier, France
}

(Received January 19, 1987; Revised May 27, 1987)

In the past decade, several arrays or lines of magnetometers have been used in order to help monitor the behaviour of external current systems. In most cases, the basic approach consists in fitting model currents with adjustable parameters to the data, a technique which has the severe disadvantage of restricting the number of parameters allowed to vary. Consequently, it is common practice to approximate the induced fields, which always contaminate ground-based magnetic measurements, by those of a perfect conductor located at some depth below the surface of the earth, this type of representation having the advantage of being solved extremely easily (even in a spherical earth) by image theory. Many careful semi-quantitative analyses of the region in terms of its electrical properties are usually carried out before the data recorded are investigated for their external contribution (e.g. KUPPERS et al., 1979), but it may happen that the depth to the equivalent perfect conductor is arbitrarily fixed without consideration of the source field. This latter procedure is never justifiable since the depth is dependent on the frequency and - to a lesser extent - on the wavenumber content of the source as well as on the average electrical properties of the region. It is also worth remembering that the definition of a single perfect conductor, fixed at a given depth during the model fitting procedure, implies that the source consists of a single dominant periodicity. A random event, such as a substorm, contains a broad spectrum of energy and may have to be analysed by initially passing the data through a narrow band filter before the calculation is done.

The determination of the depth at which to locate the perfect conductor so that it will generate field amplitudes similar to those observed at the surface of the real earth is always easy to accomplish once the periodicity content of the source is determined (e.g. see Fig. 1, after MARESCHAL, 1981). However, it is essential to remember that, since the image of a real source field by a perfect conductor is real, this representation loses all phase information. In order to evaluate the severity of the simplification, MARESCHAL (1976) compared synthetic electrojet fields induced in a flat earth either of finite conductivity varying with depth alone or of perfect conductivity. She showed that for an electrojet oriented along the E-W $(Y)$ axis, phases (or time lags as she put it) over a typical section were totally negligible for the $X$-component but more important for the $Z$-component. Depending on the location of the observer, the

*Now at IREM, Ecole Polytechnique, C.P. 6079, Succ. "A", Montreal, Canada H3C 3A7. 


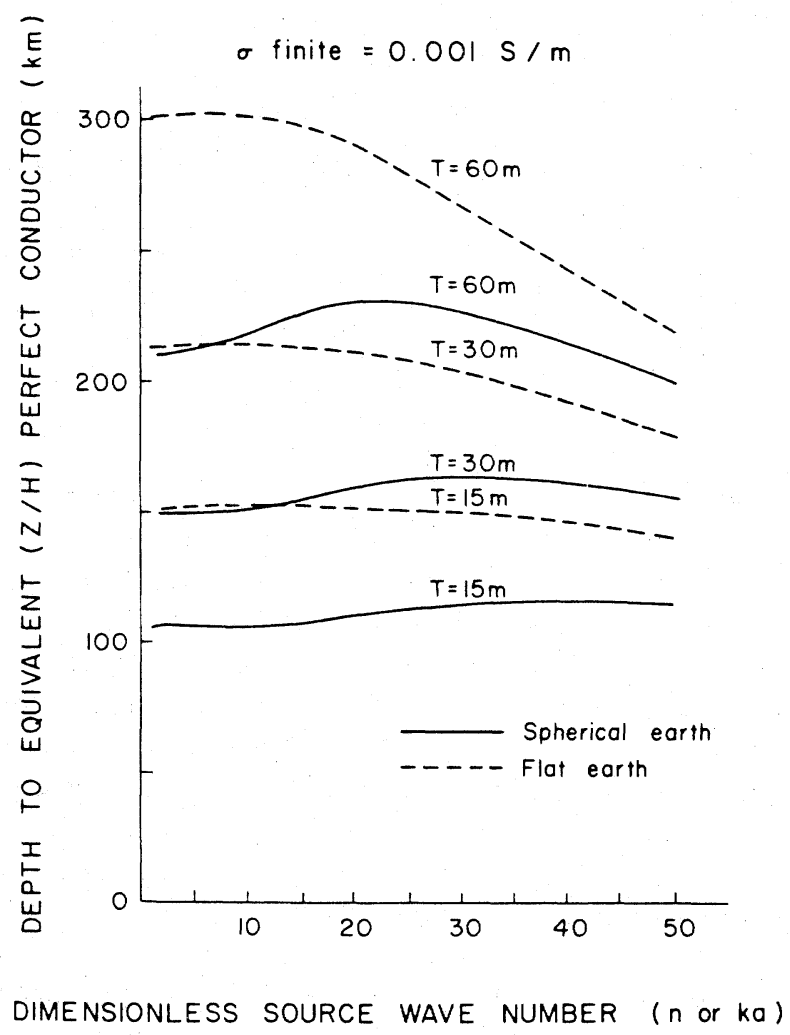

Fig. 1. Variation, with source wave-number and periodicity, of the depth of the perfect conductor equivalent to an homogeneous earth $\left(\sigma=10^{-3} \mathrm{~S} / \mathrm{m}\right)$. The amplitudes of $Z / H$ are matched at the surface (after MARESCHAL, 1981).

$Z$-component time lags could certainly reach one tenth of the source period (for 1 $\min <T<1 \mathrm{~h}$ ). She noted, however, that the larger time lags corresponded to fields recorded far from the electrojet and thus of small amplitude. Mareschal concluded that, provided the dominant periodicities of the field were taken into consideration, the representation of the earth in terms of a perfect conductor for monitoring external current systems was indeed an acceptable approximation.

The problem that we want to address here is that of magnetic variations recorded over a laterally heterogeneous region, perhaps the most extreme example being a magnetic observatory located near the coastline. In order to obtain some idea of the time lags to expect in that situation, we have extended Mareschal's work to the case of a heterogeneous superficial layer represented by a rectangular island $\left(\sigma_{\mathrm{i}}=10^{-3} \mathrm{~S} / \mathrm{m}\right)$ surrounded by a $1 \mathrm{~km}$ deep ocean $\left(\sigma_{\mathrm{o}}=1 \mathrm{~S} / \mathrm{m}\right)$. The water conductance used in this model, $1000 \mathrm{~S}$, is smaller than that of a typical ocean (e.g. $3 \mathrm{~km} \times 3 \mathrm{~S} / \mathrm{m}=9000 \mathrm{~S}$ ) but is 
selected to generate responses which would be valid near coastlines, possibly near inland heterogeneities, and would not invalidate the principles of our calculations at the periods considered. Because these calculations are based on the modelling of surface thin sheets, we cannot show how deeper conductivity contrasts would affect the results. However, the limitation is not too severe since, at the periodicities that we consider, the vast majority of lateral heterogeneities (in electrical conductivity) are found in the surface layers of the earth.

Our model consists of an electrojet source (width $=500 \mathrm{~km}$ ) flowing $115 \mathrm{~km}$ above a rectangular island $(800 \times 900 \mathrm{~km})$ as is shown in Fig. 2(a). Two periods are considered, 15 min and $1 \mathrm{~h}$, in order to make the comparison with Mareschal's earlier results more straightforward. The "normal" earth section is given in Fig. 2(b) and consists of 3 homogeneous layers $\left(\rho_{\mathrm{i}}=10^{3} \Omega \mathrm{m}, h_{1}=1 \mathrm{~km} ; \rho_{1}=10^{4} \Omega \mathrm{m}, h_{2}=30 \mathrm{~km}\right.$; $\left.\rho_{2}=10^{2} \Omega \mathrm{m}\right)$. The calculations are based on the thin sheet model of VASSEUR and WEIDELT (1977) for an electrojet source similar to that presented by MARESCHAL et al. (1987) to simulate the daytime induction arrows observed in the region of southern India directly beneath the equatorial electrojet. Note that substorm fields are due to very dynamic spatial and amplitude variations both of the auroral electrojets and of the associated field-aligned currents and that truly realistic modelling would require the use of 3-dim sources and of spherical geometry (e.g. MARESCHAL, 1986). Unfortunately, the induction problem would then become quite complex and the induced fields would have to be approximated by the response of simplistic earth models. Since it is the aim of this paper to give some idea of the error introduced when the most simplistic of these earth models is used to simulate the effect of real induced currents, we have decided to simplify the problem by considering a region small enough to permit a flattening of the earth and by neglecting the effects of field-aligned currents, which, in this approximation, become vertical. Note, finally, that the example of surface heterogeneity selected for this study is well suited to the thin sheet algorithm that we use and which requires the anomalous structure (here, the island) to be entirely surrounded by a homogeneous structure extending to infinity. Thus, the close proximity of the boundaries of our study area to the island does not affect the validity of our results.

Without lateral heterogeneities in the first layer, the surface electrojet field has only two components, $B_{x}$ and $\boldsymbol{B}_{z}$. The corresponding time lags are given in Fig. 3 for periods of 900 and $3600 \mathrm{~s}$ (a negative time lag corresponds to an advanced field). They are quite similar to those presented by MARESCHAL (1976), the slight difference being due to the simpler layering that we consider here (3 layers instead of 5). However, as soon as lateral discontinuities are introduced within the first layer, currents start to deviate and a $B_{y}$ component is created. The time lags in the three components are affected by their distance to the electrojet, their distance to the coastlines, and the angle between the coast direction and that of the source polarization.

The time lags corresponding to $B_{x}\left(t_{x}\right)$ reach larger amplitudes than those calculated over a laterally homogeneous layered section (LHLS). Here, two effects are competing. One is due to the electrojet itself which generates bands of positive lags (in a background of negative lags) beyond its northern and southern edges. These 


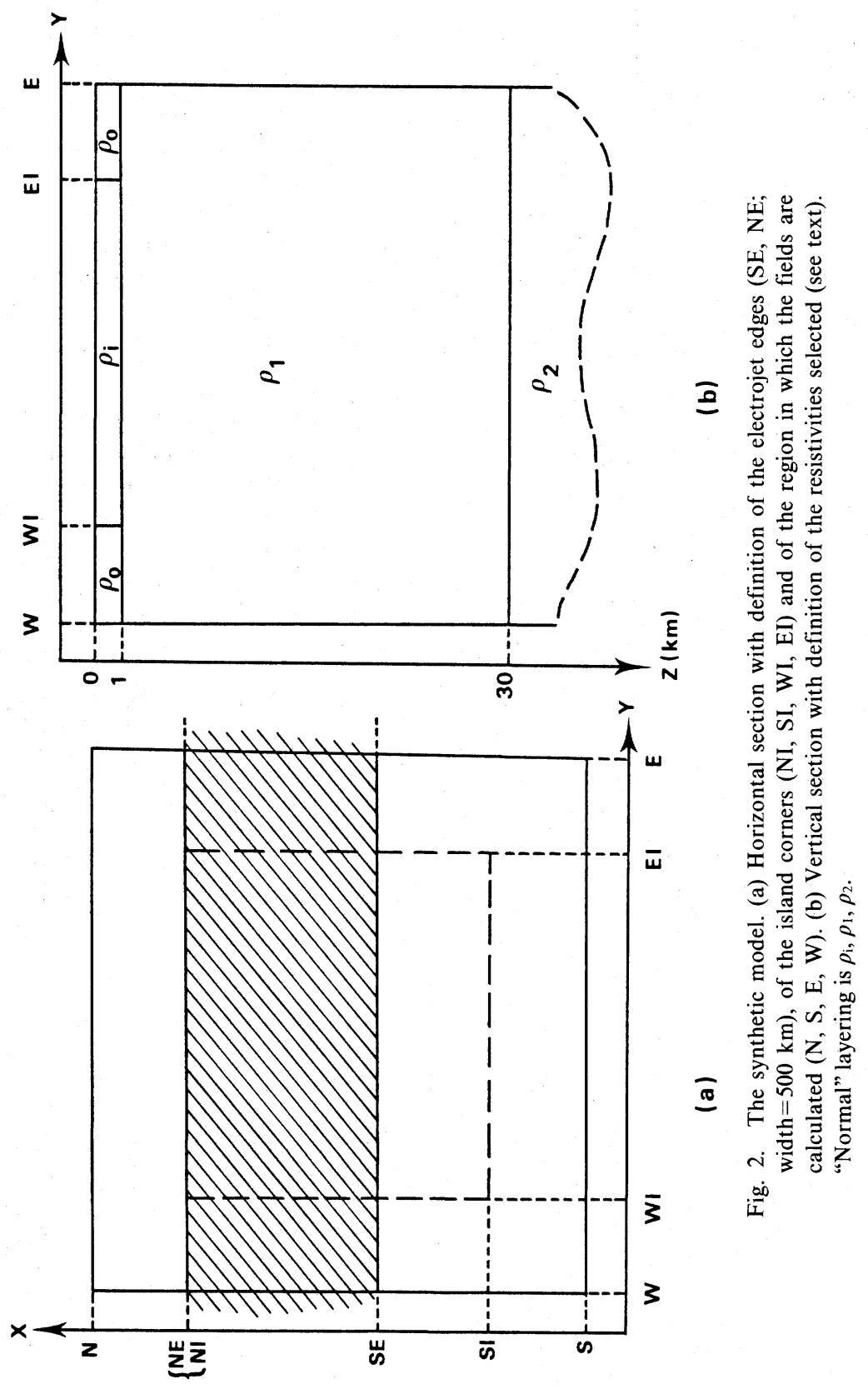




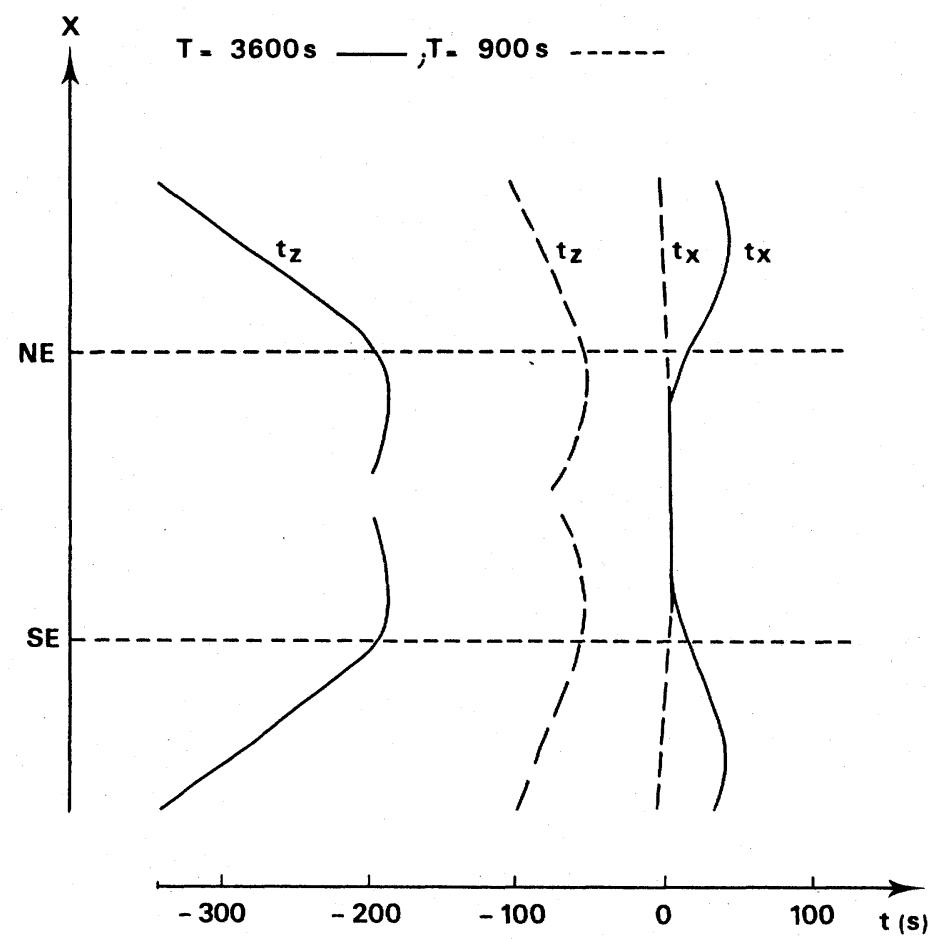

Fig. 3. Variations of time lags $(t)$ calculated over the laterally homogeneous section (LHLS) defined as "normal" in Fig. 2(b). The lags vary with distance to the electrojet and with source period.

bands become progressively larger and better defined as the periodicity of the source increases. The effect is most clearly recognized in Fig. 3, where it is not coupled to any surface heterogeneity effect. It indicates a maximum $t_{x}$ of $48 \mathrm{~s}$ at $200 \mathrm{~km}$ from the edges for $T=3600 \mathrm{~s}$ whereas the region of positive lags is barely apparent at $900 \mathrm{~s}$. The second effect is that of the conductivity discontinuity. It is responsible for introducing positive lags on the ocean side (better conductor) and negative lags on the other side. Figure 4 indicates that, as it could be expected, the electrojet edge effect remains more important at $3600 \mathrm{~s}$ than at $900 \mathrm{~s}$ over a section including surface heterogeneities. In fact, at $900 \mathrm{~s}$, it is almost balanced by the coast effect which is responsible for the very small positive lags shown to the east and west of the island $\left(t_{x} \simeq 1 \mathrm{~s}\right)$. At this period, most lags are very small (less than $1 \% T$ ), except at the northern and southern coastline where they, nonetheless, remain inferior to $3 \% T$. They reach $4 \% T$ at the same location for $T=3600 \mathrm{~s}$. Thus, and even though the $900 \mathrm{~s}$ lags of Fig. 4(b) may include some numerical artifact due to the very small value of their individual components, the N-S behaviour of $t_{x}$ in Figs. 4(a) and (b) follow the trend observed over the LHLS (Fig. 3) (remember that the bands of larger lags obtained near the island edges are in the ocean). 


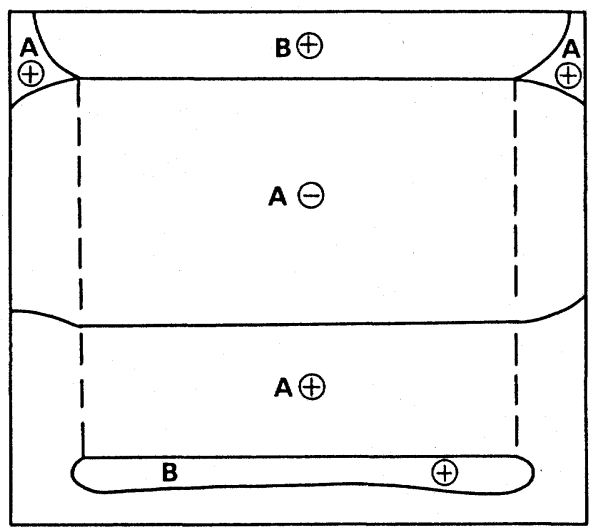

( a )

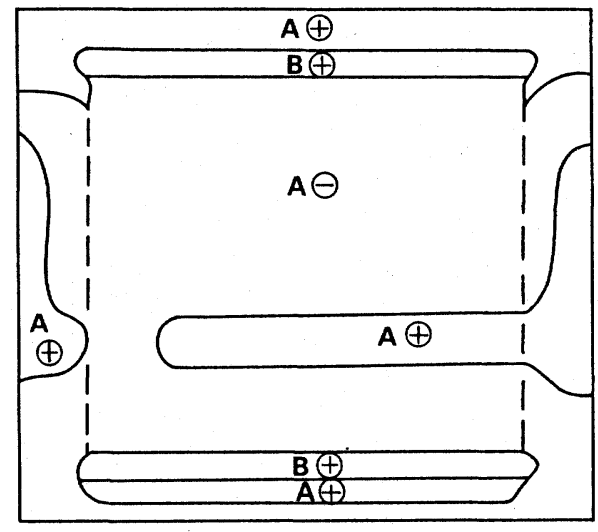

(b)

Fig. 4. $t_{x}$ for laterally heterogeneous model (a) with $T=3600 \mathrm{~s},(\mathrm{~b})$ with $T=900 \mathrm{~s}$. $\oplus$ is for a positive lag, $\ominus$ is for a negative lag (thus field advance). The amplitude of the lag is defined by the lettering: "A" $=t<2 \% T, " \mathrm{~B} "=2 \% \leqslant t<4 \% T, " \mathrm{C} "=4 \% \leqslant t<6 \%, " \mathrm{D} "=6 \% \leqslant t<10 \% T, " \mathrm{E} "=t \geqslant 10 \% T$. The dashed line gives the outline of the island.

The $Z$-component is also characterized by relatively well behaved time lags. To the typical smooth variation with latitude calculated over the LHLS is added the coast effect which tends to decrease systematically the amplitude of $t_{z}$. The relative minima observed at the edges of the electrojet (see Fig. 3) are enhanced by the proximity of the coast, especially, on the northern border of our synthetic model. The maximum lag amplitude recorded at $900 \mathrm{~s}$ is $56 \mathrm{~s}$ as opposed to $98 \mathrm{~s}$ for the LHLS. Thus, in terms of the $Z$-component alone, the representation of the island by some perfect conductor (at the appropriate depth) is more justifiable than it is over the LHLS. Note, however, that the time lags calculated over the ocean increase progressively up to and even slightly above their LHLS value as the distance from the island increases. Again, this behaviour is frequency dependent: $250 \mathrm{~km}$ north of the northern coastline, the average $t_{z}(900 \mathrm{~s})$ reaches $-93 \mathrm{~s}$ against $-89 \mathrm{~s}$ for the LHLS, whereas the same average $t_{z}(3600 \mathrm{~s})$ only reaches $-231 \mathrm{~s}$ against $-287 \mathrm{~s}$ for the LHLS.

A significant difference from Mareschal's earlier results comes from the existence of a $Y$-component (perpendicular to the source field polarization). The amplitude of the component itself is always quite small compared to that of $B_{x}\left(B_{y} \max \leqslant 1 / 10 B_{x}\right.$ $\max )$, but its phase and time lags are quite variable and usually very large ( $t_{y}$ can easily reach $20 \% T$ ). They tend to be negative at the corners of the island and at the "front" of the conductivity contrast where currents deviate and charges build up. They are negative again roughly along the N-S line of symmetry of the island. However, the variability of individual values is so large (because we are dealing with ratios of so small quantities) that it does not warrant the presentation of detailed results. For instance, at $T=3600 \mathrm{~s}, t_{y}$ values range from $-882 \mathrm{~s}$ to $+859 \mathrm{~s}$. Note that the 


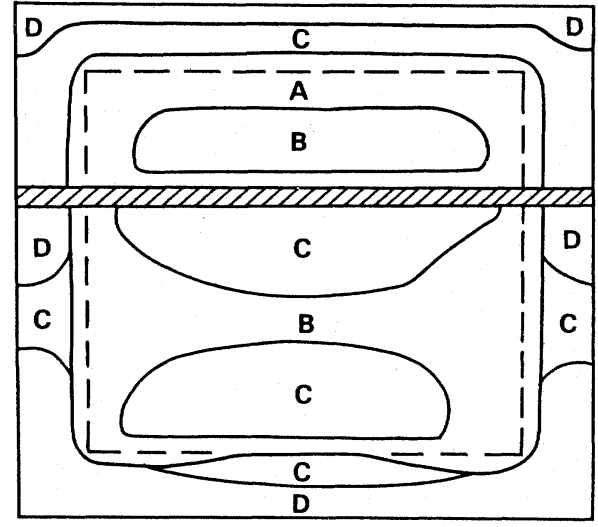

(a)

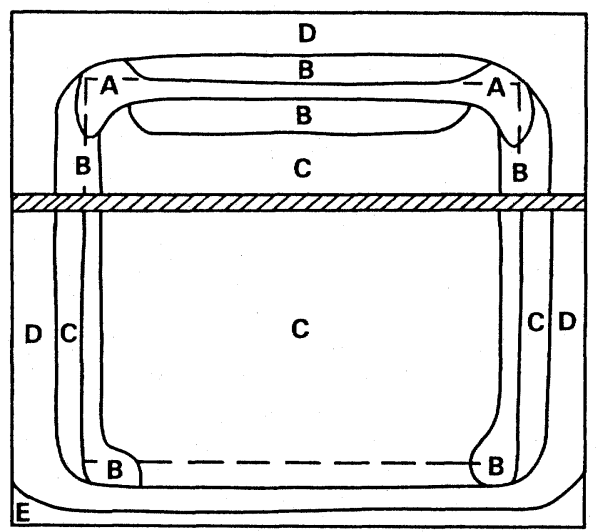

(b)

Fig. 5. Same as Fig. 4 except for $t_{z}$. All lags are negative. The dashed E-W region is the centre of the electrojet.

$Y$-component of our model field is entirely due to current deviation, which enhances the imaginary contribution and thus produces large $t_{z}$ 's. A real substorm field will include contributions from field-aligned currents and thus normal $Y$-components which, depending on the event and on the observation point, may occasionally become as large as the $X$-component (e.g. RostOKER and HUGHES, 1979). This situation would be rather extreme, but indicates that the presence of normal $B_{y}$ 's in any substorm field will help reduce the time lags presented here. However, because of the restrictive type of source that we selected in this study, a more explicit analysis of $t_{y}$ is not possible.

In conclusion, and in so far as simple model sources can be used to test the responses of various model earths to complex sources, it does not appear that lateral heterogeneities in the upper layer render the representation of the induced field by that of a perfect conductor more invalid than it is in the case of a normally layered earth. This conclusion should hardly be considered as supportive of any oversimplified model fitting approach when it can be avoided. Representing the earth induction effects by those of a perfect conductor is always a first-order approximation and is only justified when the depth to this equivalent conductor is calculated in function of the periodicity content of the source. Statistical methods should be used in conjunction with parameter adjustments as often as possible in order to separate internal and external contributions. The recent work of RICHMOND and BAUMJOHANN (1984) is certainly quite promising in that respect.

Our grateful thanks go to Ian Ferguson and to Alan Jones for their constructive comments, which definitely helped improve the paper. 


\section{REFERENCES}

Kuppers, F., J. Untiedt, W. Baumjohann, K. Lange, and A. G. Jones, A two-dimensional magnetometer array for ground-based observations of auroral zone electric currents during the international magnetospheric study (IMS), J. Geophys., 46, 429-450, 1979.

MARESCHAL, M., On the problem of simulating the earth's induction effects in modelling polar magnetic substorms, Rev. Geophys. Space Phys., 14, 403-409, 1976.

MaReschal, M., Source effects and the interpretation of geomagnetic sounding data at sub-auroral latitudes, Geophys. J. R. Astr. Soc., 67, 125-136, 1981.

MARESChaL, M., Modelling of natural sources of magnetospheric origin in the interpretation of regional induction studies: a review, Surveys Geophys., 8, 261-300, 1986.

Mareschal, M., B. J. Srivastava, G. Vasseur, and B. P. Singh, Induction models of southern India and the effect of off-shore geology, Phys. Earth Planet. Int., 45, 137-148, 1987.

Richmond, A. D. and W. BAumjohann, Three-dimensional analysis of magnetometer array data, $J$. Geophys., 54, 138-156, 1984.

Rostoker, G. and T. J. Hughes, A comprehensive model current system for high-latitude magnetic activity, II: The substorm component, Geophys. J. R. Astr. Soc., 58, 571-581, 1979.

VAsseur, G. and P. Weidelt, Bimodal electromagnetic induction in non-uniform thin sheets with an application to the northern Pyrenean induction anomaly, Geophys. J. R. Astr. Soc., 51, 669-690, 1977. 